\title{
Cellular and Molecular Mechanisms of Hepatic Fibrosis
}

\author{
Minling Cao*, Xiaoling Chi, Junmin Jiang and Guangjun Tian \\ Department of Hepatology, Guangdong Province Hospital of TCM, Guangzhou, Guangdong 510007, China
}

\begin{abstract}
The occurrence of hepatic fibrosis is a multi-factor involved process. The key is the activation of hepatic stellate cells (HSC). Synthesis of extracellular matrix in the liver cells increases while degradation decreases. This paper reviews the tissue factors and the mechanism closely related to the forming of hepatic fibrosis.
\end{abstract}

\section{KEYWORDS}

Hepatic fibrosis

Hepatic stellate cells

Mechanism

\section{Introduction}

Advanced liver fibrosis results in cirrhosis, liver failure and portal hypertension. Liver transplantation is often needed for patients with end-stage liver disease, including collagen, Liver fibrosis is the excessive accumulation of extra-cellular matrix proteins that occurs in most types of chronic liver diseases. With the research in recent years, the knowledge of the cellular and molecular mechanisms of liver fibrosis has greatly advanced. Activated hepatic stellate cells, portal fibroblasts, myofibroblasts, bone marrow cells have been identified as major collagen-producing cells in the injured liver [1].

\section{Historical perspective}

Hepatic fibrosis was thought to be a passive and irreversible process due to the collapse of hepatic parenchyma and its substitution with a collagen-rich tissue in history. Currently, it is considered as a model of the wound-healing response to chronic liver injury. Rapid and slower fibrosis were identified, and genetic and environmental factors influencing fibrosis progression were partially uncovered.

\footnotetext{
Copyright (c) 2015 Minling Cao et al.

doi: $10.18686 /$ aem.v4i3.4

Received: August 9, 2015; Accepted: September 2, 2015; Published online: September 18, 2015

This is an open-access article distributed under the terms of the Creative Commons Attribution Unported License (http://creativecommons.org/ licenses/by-nc/4.0/), which permits unrestricted use, distribution, and reproduction in any medium, provided the original work is properly cited.

${ }^{\star}$ Corresponding author: Department of Hepatology, Guangdong Province Hospital of TCM, Guangzhou, Guangdong 510007, China. E-mail: 418594787@qq.com
}

The main causes of liver fibrosis in industrialized countries include chronic HBV infection, chronic HCV infection, alcohol abuse and nonalcoholic steatohepatitis (NASH). Previous studies considered the accumulation of ECM proteins, which results in chronic damage to the liver, which is a characteristic of most types of chronic liver diseases. The accumulation of ECM proteins distorts the hepatic architecture by forming a fibrous scar, and the subsequent development of nodules of regenerating hepatocytes defines cirrhosis. Cirrhosis produces hepatocellular dysfunction and increased intrahepatic resistance to blood flow, resulting in hepatic insufficiency and portal hypertension, respectively [2-4].

\section{Natural history and diagnosis}

The onset of liver fibrosis is usually insidious, and most of the related morbidity and mortality occur after the development of cirrhosis. In the majority of patients, progression to cirrhosis occurs after an interval of 15-20 years. Major clinical complications of cirrhosis include ascites, renal failure, hepatic encephalopathy, and variceal bleeding. Patients with cirrhosis can remain free of major complications for several years (compensated cirrhosis). Decompensated cirrhosis is associated with short survival, and liver transplantation is often the only effective therapy. Cirrhosis is also a risk factor for developing hepatocellular carcinoma [5].

\section{Pathogenesis of liver fibrosis}

Hepatic fibrosis is the result of the wound-healing response of the liver to repeated injury. After an acute liver injury, 
parenchymal cells regenerate and replace the necrotic or apoptotic cells. This process is associated with an inflammatory response and a limited deposition of ECM. If the hepatic injury persists, eventually the liver regeneration fails, and hepatocytes are substituted with abundant ECM, including fibrillar collagen. The distribution of this fibrous material depends on the origin of the liver injury. In chronic viral hepatitis and chronic cholestatic disorders, the fibrotic tissue is initially located around portal tracts, while in alcohol-induced liver disease, it locates in pericentral and perisinusoidal areas. With the progress of fibrotic liver diseases, disease progresses from collagen bands to bridging fibrosis to cirrhosis occurs. Hepatic cell types other than HSCs may also have fibrogenic potential. Myofibroblasts derived from small portal vessels proliferate around biliary tracts in cholestasis-induced liver fibrosis to initiate collagen deposition. HSCs and portal myofibroblasts differ in specific cell markers and response to apoptotic stimuli. $\mathrm{CD}^{34+} \mathrm{CD}^{38-}$ hematopoietic stem cells with various growth factors have been shown to generate HSCs and myofibroblasts of bone marrow origin that infiltrate human livers tissue remodeling. These data suggest that cells originating in bone marrow can be a source of fibrogenic cells in the injured liver. The relative importance of each cell type in liver fibrogenesis may depend on the origin of the liver injury $[6,9]$.

Liver fibrosis is associated with major alterations in both the quantity and composition of ECM. In advanced stages, the liver contains approximately 6 times or above of ECM than normal, including collagens, fibronectin, elastin, laminin, hyaluronan, and proteoglycans. Accumulation of ECM results from both increased synthesis and decreased degradation. Decreased activity of ECMremoving MMPs is mainly due to an overexpression of their specific inhibitors (TIMPs). HSCs are the main ECM-producing cells in the injured liver. In the normal liver, HSCs reside in the space of Disse and are the major storage sites of vitamin A. Following chronic injury, HSCs activate or transdifferentiate into myofibroblast-like cells, acquiring contractile, proinflammatory, and fibrogenic properties Activated HSCs migrate and accumulate at the sites of tissue repair, secreting large amounts of ECM and regulating ECM degradation. PDGF, mainly produced by Kupffer cells, is the predominant mitogen for activated HSCs. Collagen synthesis in HSCs is regulated at the transcriptional and post-transcriptional levels. Increased collagen mRNA stability mediates the increased collagen synthesis in activated HSCs. In these cells, posttranscriptional regulation of collagen is governed by sequences in the $3^{\prime}$ untranslated region via the RNAbinding protein $\mathrm{CP} 2$ as well as a stem-loop structure in the 5 ' end of collagen mRNA. Quiescent HSCs express markers that are characteristic of adipocytes (PPAR, SREBP-1c, and leptin), while activated HSCs express myogenic markers (smooth muscle actin, c-myb, and myocyte enhancer factor-2) $[7,8]$.

\section{Key cytokines involved in liver fibrosis}

Cytokines regulate the inflammatory response to injury modulate hepatic fibrogenesis in vivo and in vitro. Monocyte chemotactic protein type 1 and RANTES stimulate fibrogenesis while IL-10 and IFN exert have the opposite effect. Among growth factors, TGF- $\beta 1$ appears to be a key mediator in human fibrogenesis. HSCs, TGF- $\beta 1$ favors the transition to myofibroblast-like cells, stimulates the synthesis of ECM proteins, and inhibits their degradation. PDGF is the most potent mitogen for HSCs and is rised in the fibrotic liver; its inhibition attenuates experimental liver fibrogenesis. Adipokines, which are cytokines mainly derived from the adipose tissue, regulate liver fibrogenesis. Leptin is required for HSC activation and fibrosis development. In contrast, adiponectin markedly inhibits liver fibrogenesis in vitro and in vivo. The actions of these cytokines may explain why obesity influences fibrosis development in patients with chronic hepatitis $\mathrm{C}$ [10].

Cytokines with vasoactive properties also regulate liver fibrogenesis. Vasodilator substances (such as nitric oxide, relaxin) exert antifibrotic effects while vasoconstrictors (such as norepinephrine, angiotensin II) have opposite effects. Endothelin-1, a powerful vasoconstrictor, stimulates fibrogenesis through its type A receptor in vasoactive cytokines, angiotensin II seems to play a major role in liver fibrogenesis. Angiotensin II is the effector peptide of the renin-angiotensin system, which is a major regulator of arterial pressure homeostasis in humans. Key components of this system are locally expressed in chronically injured livers, and activated HSCs generate angiotensin II importantly, pharmacological and/or genetic ablation of the renin-angiotensin system markedly attenuates experimental liver fibrosis. Angiotensin II induces hepatic inflammation and stimulates an array of fibrogenic actions in activated HSCs, including cell proliferation, cell migration, secretion of proinflammatory cytokines, and collagen synthesis. Unlike the phagocytic type, NADPH oxidases present in fibrogenic cell types are constitutively active, producing relatively low levels of ROS under basal conditions and generating higher levels of oxidants in response to cytokines, stimulating redoxsensitive intracellular pathways. NADPH oxidase also plays a key role in the inflammatory actions of Kupffer cells $[11,12]$.

\section{Conclusion and future directions}

Fibrosis is an excessive wound healing response that occurs in most forms of chronic liver disease and results in the deposition of scar tissue, i.e., excess extracellular matrix (ECM). With ongoing liver damage, fibrosis may progress to cirrhosis, which is characterized by a distortion of the liver vasculature and architecture, and is the major 
determinant of morbidity and mortality in patients with liver disease. However, many patients either do not respond to causal treatment or are diagnosed with advanced fibrosis or cirrhosis. Because fibrosis and especially cirrhosis are the major predictors of liver-related morbidity and mortality, there is an urgent need to develop, test, and monitor antifibrotic treatments, in order to prevent, halt, or even reverse liver fibrosis or cirrhosis. Although we have made impressive progress in our understanding of the mechanisms based on the pathogenesis of liver fibrosis in the past two decades, translation of this knowledge into antifibrotic therapies has ground to a halt short of clinical trials.

\section{Acknowledgments}

I would like to deeply thank to the help of my advisors, Professors Chi Xiaoling and Jiang Junmin.

\section{Conflicts of interest}

These authors have no conflicts of interest to declare.

\section{Authors' contributions}

These authors contributed equally to this work.

\section{Reference}

1. PanJJ, Susan PF, Chen CR, Ariel EF, Joseph BMC, Rahbar MH, Laura B, Michael BF. Burden of nonalcoholic fatty liver disease and advanced fibrosis in a Texas Hispanic community cohort. World Journal of Hepatology. 2015;11:1586-1594.

2. Lin Z, Sun YL, Shi WL, Ma X, Chen Z, Wang JB, Li RS, Song $X A$, Liu HH, Zhao YL, Xiao XH. Protective effect of fu-qi granule on carbon tetrachloride-induced liver fibrosis in rats. World Journal of Pharmacology. 2015;02:227-235.

3. Zhen YZ, Li NR, He HW, Zhao SS, Zhang GL, Hao XF, Shao RG. Protective effect of bicyclol against bile duct ligation-induced hepatic fibrosis in rats. World Journal of Gastroenterology. 2015;23:7155-7164.

4. Xing ZZ, Huang LY, Wu CR, You H, Ma H, Jia JD. Activated rat hepatic stellate cells influence Th1/Th2 profile in vitro. World Journal of Gastroenterology. 2015;23:71657171.

5. Shen QL, Chen YJ, Wang ZM, Zhang TC, Pang WB, Shu $\mathrm{J}$, Peng $\mathrm{CH}$. Assessment of liver fibrosis by Fibroscan as compared to liver biopsy in biliary atresia. World Journal of Gastroenterology. 2015;22:6931-6936.

6. Adriana MS, Luis D HO, Martha S LL, Juan AB. New gene therapy strategies for hepatic fibrosis. World Journal of Gastroenterology. 2015;13:3813-3825.

7. Li M, Wang XF, Shi JJ, Li YP, Yang N, Zhai S, Dang ShuangSuo. Caffeic acid phenethyl ester inhibits liver fibrosis in rats. World Journal of Gastroenterology. 2015;13:38933903.

8. Suk KT, Kim DJ. Staging of liver fibrosis or cirrhosis: The role of hepatic venous pressure gradient measurement. World Journal of Hepatology. 2015;03:607-615.

9. Zhai L, Qiu LY, Zu Y, Yan Y, Ren XZ, Zhao JF, Liu YJ, Liu JB, Qian LX. Contrast-enhanced ultrasound for quantitative assessment of portal pressure in canine liver fibrosis. World Journal of Gastroenterology. 2015;15:4509-4516.

10. Chang WJ, Song LJ, Yi T, Shen KT, Wang HS, Gao XD, Li M, Xu JM, Niu WX, Qin XY. Early activated hepatic stellate cell-derived molecules reverse acute hepatic injury. World Journal of Gastroenterology. 2015;14:4184-4194.

11. Thomas K, Joachim B, Nikita G, Franziska L, Harald B, Nicolas L, Alexander S, Bettina R, Rima C, Michael T, Johannes W, Volker K. Estimating steatosis and fibrosis: Comparison of acoustic structure quantification with established techniques. World Journal of Gastroenterology. 2015;16:4894-4902.

12. Santosh MS. Liver cirrhosis in hepatic vena cava syndrome (or membranous obstruction of inferior vena cava). World Journal of Hepatology. 2015;06:874-884. 